\title{
Broadband Wavelength-swept Raman Laser for Fourier-domain Mode Locked Swept-source OCT
}

\author{
Hyung-Seok Lee, Eun-Joo Jung, Myung-Yung Jeong, and Chang-Seok Kim* \\ Department of Cogno Mechatronics Engineering, Pusan National University, Pusan 609-735, Korea
}

(Received June 8, 2009 : revised August 10, 2009 : accepted September 1, 2009)

\begin{abstract}
A novel broadband wavelength-swept Raman laser was used to implement Fourier-domain mode locked (FDML) swept-source optical coherence tomography (SS-OCT). Instead of a conventional semiconductor optical amplifier, this study used broadband optical fiber Raman amplification, over $50 \mathrm{~nm}$ centered around $1545 \mathrm{~nm}$, using a multi-wavelength optical pumping scheme, which was implemented with the four laser diodes at the center wavelengths of 1425, 1435, 1455 and 1465 $\mathrm{nm}$, respectively, and the maximum operating power of $150 \mathrm{~mW}$ each. The operating swept frequency of the laser was determined to $16.7 \mathrm{kHz}$ from the FDML condition of $12 \mathrm{~km}$ optical fiber in the ring cavity. The OCT images were obtained using the novel broadband wavelengthswept Raman laser source.
\end{abstract}

Keywords: Optical coherence tomography, Fiber Raman amplifier, Wavelength-swept fiber laser

OCIS codes: (110.4500) Optical coherence tomography; (140.3550) Lasers, Raman; (060.3510) Lasers fiber

\section{INTRODUCTION}

Optical coherence tomography (OCT) employs low coherence interferometry to acquire micron-scale crosssectional images non-invasively [1-3]. Fourier-domain OCT has many advantages over conventional timedomain OCT, such as a higher acquisition speed without mechanical delay line and a higher signal-to-noise ratio. A promising method for high-speed imaging is offered by swept-source (SS) OCT [1-5]. The performance of high-speed and high-resolution SS-OCT imaging depends on the effective sweep rate and tuning of the spectral range of the wavelength-swept laser source, respectively. Generally, most wavelength-swept lasers for SSOCT employ a semiconductor optical amplifier (SOA) as the gain medium [1-4]. The typical carrier relaxation time of a SOA is several nanoseconds and its gain band normally provides a bandwidth less than $100 \mathrm{~nm}$ because the sweep rate and spectral range are limited by the carrier relaxation times and entire gain band of the gain medium, respectively. Fourier domain modelocked (FDML) techniques have been reported to increase the sweeping speed of wavelength-swept lasers using

*Corresponding author: ckim@pusan.ac.kr additional delayed length of optical fiber in the laser cavity [2]. However, the long length of optical fiber over $\sim \mathrm{km}$ induces additional insertion loss of the cavity, which is unhelpful for lasing operations. Recently, we proposed that an additional delayed length of an optical fiber can be used as a gain medium instead of a loss medium when fiber Raman amplification is generated by a high power pump light [6].

In this study, a novel broadband wavelength-swept Raman laser was demonstrated using a multi-wavelength optical pumping scheme to implement FDML SS-OCT imaging. This is the first experimental demonstration of a broadband SS-OCT based on a fiber Raman amplifier as a broad gain medium over $50 \mathrm{~nm}$, compared with the previous report of narrower Raman gain using a single-wavelength optical pumping scheme [6,7]. As high power optical pump sources have become commercially available, a fiber Raman amplification based on stimulated Raman scattering in silica optical fibers has been studied widely, particularly in wavelength-division-multiplexed (WDM) telecommunication system applications [8-13]. The fiber Raman amplifier has several advantages, such as low noise, arbitrary gain band determined on the band of the optical pump sources, high temperature stability and instantaneous carrier relaxation times of 
the order of several femto-seconds for silica [9]. It is expected that the application of a fiber Raman amplifier can be extended to biomedical imaging based on these experimental results due to the increasing demand for higher-speed and a wider-gain band for the real time and high resolution OCT imaging, respectively.

\section{WAVELENGTH SWEPT RAMAN LASER}

Figure 1 shows a schematic diagram of the wavelengthswept laser based on fiber Raman amplifier gain. The laser consisted of a $12 \mathrm{~km}$ Raman gain optical fiber, a wavelength division multiplexing (WDM) coupler to receive a multi-wavelength optical pump power, two isolators for a unidirectional configuration, a polarization controller (PC), fiber Fabry-Perot tunable filter (FFP-TF) and an output coupler into a SS-OCT system. For an efficient and stable Raman gain process, a standard $11 \mathrm{~km}$ dispersion shifted fiber (DSF) and a 1 $\mathrm{km}$ dispersion compensation fiber (DCF) are optimally used for the Raman gain optical fiber medium because a normal dispersion scheme is preferred in the gain wavelength region of approximately $1500 \sim 1600 \mathrm{~nm}$. When the zero dispersion wavelength, $\lambda_{0}$, of $11 \mathrm{~km}$ DSF is approximately $1550 \mathrm{~nm}$, the total dispersion value of the $12 \mathrm{~km}$ gain media will take the normal dispersion characteristics over the $1550 \mathrm{~nm}$ region with the aid of a $1 \mathrm{~km}$ DCF [8]. A 14XX/15XX WDM coupler was used in the ring laser cavity for the effective coupling of the multi-wavelength pumping light below $1500 \mathrm{~nm}$ and the round-trip signal light over $1500 \mathrm{~nm}$.

A broadband Raman gain region is needed for higher resolution OCT imaging. In this study, a pump combining coupler was used to generate a multi-wavelength pumping scheme with four laser diodes. The center wavelength of each LD was 1425, 1435, 1455 and $1465 \mathrm{~nm}$,

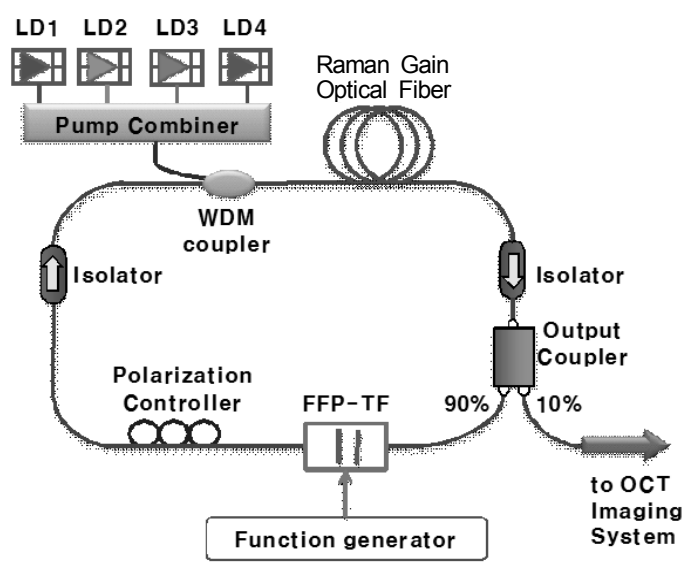

FIG. 1. Setup of wavelength-swept laser including the Raman amplifier based on multi-wavelength pumping scheme (LD: Laser Diode, FFR-TF: Fiber Fabry-Perot Tunable Filter). respectively, and the maximum operating power was $150 \mathrm{~mW}$ for each LD. Fig. 2 shows a spectral comparison of the Raman amplifier; in each case when LD 1 was turned on, LD 1 and 2 were turned on, and LD 1, 2, 3 , and 4 were turned on, respectively. The results show that the wider pumping wavelength induces a broader Raman gain wavelength region.

Considering the interaction between the pump and Stokes waves to find the Raman threshold, the evolution of the signal and pump power levels can be described using the following coupled equations [11],

$$
\begin{aligned}
& \frac{d I_{s}}{d z}=g_{R} I_{p} I_{s}-\alpha_{s} I_{s} \\
& \frac{d I_{p}}{d z}=-\frac{\omega_{p}}{\omega_{s}} g_{R} I_{p} I_{s}-\alpha_{p} I_{p}
\end{aligned}
$$

where $I_{s}$ is the Stokes intensity, $I_{p}$ is the pump intensity, $g_{R}$ is the Raman-gain coefficient and the absorption coefficients $\alpha_{s}$ and $\alpha_{p}$ denote the fiber loss at the Stokes and pump frequencies. The first terms on the right side of each equation describe the nonlinear stimulated process and couple the two waves. The second terms on the right side are due to the linear absorption in the fiber medium. From a numerical simulation of the cascaded Raman frequency shift $[10,11]$, it was confirmed that a wider distribution of multi-wavelength pumping below $1500 \mathrm{~nm}$ will reduce the broader distribution of Raman gain above the 1500 $\mathrm{nm}$ region.

A novel broadband wavelength-swept FDML Raman laser was demonstrated based on the broadband Raman gain amplification. Considering the length of a $12 \mathrm{~km}$ Raman gain fiber, the operating frequency of a fiber Fabry-Perot tunable filter was determined to be 16.7 $\mathrm{kHz}$ under the FDML conditions [2]. Fig. 3 (a) shows the output spectrum of a Raman laser at $1553.3 \mathrm{~nm}$

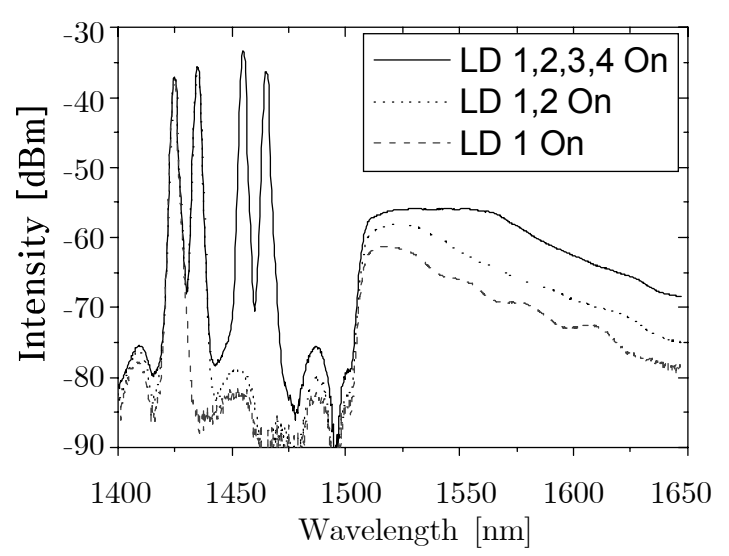

FIG. 2. Comparison of various spectra of Raman amplifier when LD 1 is on, LD 1 and 2 are on, and LD 1,2,3, and 4 are on, respectively. 


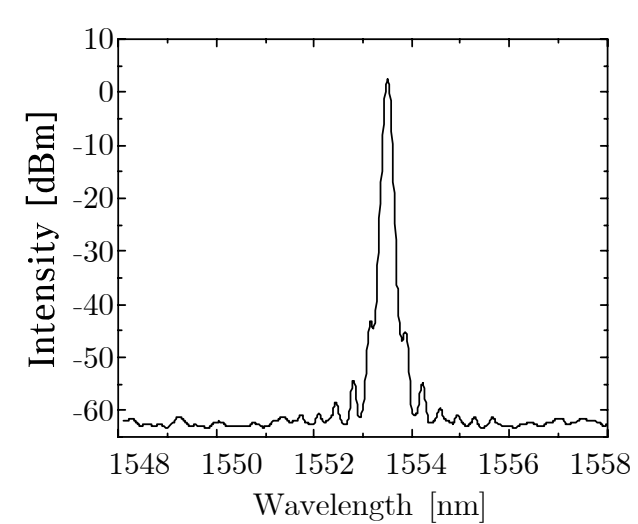

(a)

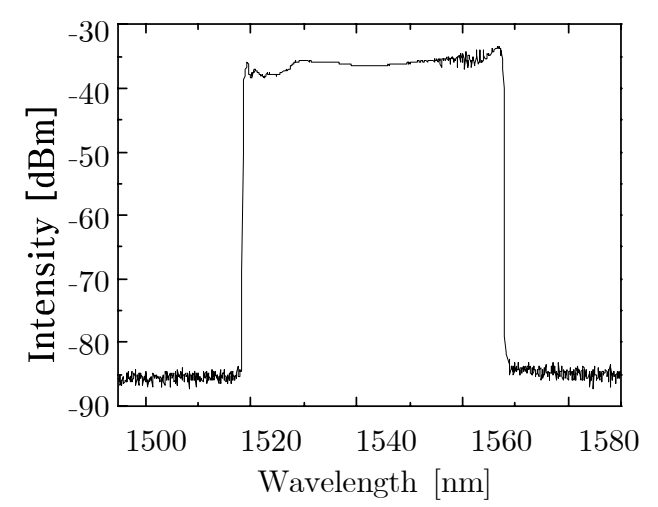

(b)

FIG. 3. (a) Output spectrum of Raman laser when FFP-TF is not sweeping, (b) Output spectrum in peak hold mode of the wavelength swept Raman laser when FFP-TF is sweeping repeatedly.

when the FFP-TF was not sweeping. The results show that the line-width is $0.07 \mathrm{~nm}$ and the signal-to-noise of the laser is larger than $60 \mathrm{~dB}$. Fig. 3 (b) shows the measurement results of the overall output spectrum of the wavelength swept Raman laser when the FFP-TF is sweeping at a $16.7 \mathrm{kHz}$ sinusoidal frequency. At the peak hold mode of the optical spectrum analyzer (OSA), the $3 \mathrm{~dB}$ bandwidth of $50 \mathrm{~nm}$ was centered at approximately $1543 \mathrm{~nm}$ and the extinction ratio was approximately $50 \mathrm{~dB}$. Compared to the single wavelength pumping for the $30 \mathrm{~nm}$ bandwidth [7], the bandwidth of the sweeping range was almost doubled by the suggested multi-wavelength pumping schemes.

\section{OCT IMAGING}

Figure 4 shows the experimental setup for OCT imaging based on the wavelength swept Raman laser. Two tunable directional coupler and two circulators were used to construct a Mach-Zehnder interferometer. Using the tunable directional coupler, the optical power between the sample port and reflecting mirror port were controlled manually.

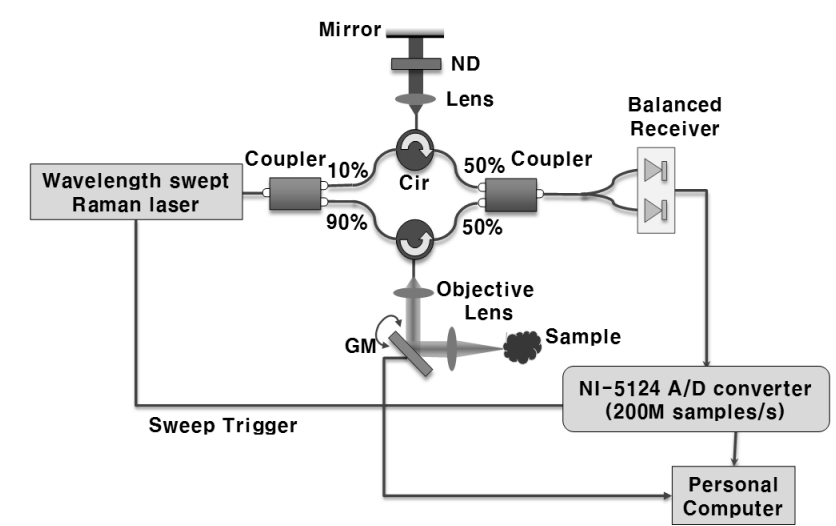

FIG. 4. Scheme of experimental setup for OCT imaging (ND: Neutral Density Filter, GM: Galvano Mirror).

The sample path was scanned laterally using the galvano mirror and objective lens. A cross sectional image was produced by transversely scanning the beam across the sample while collecting a reflecting profile at each point. The converted reflecting intensities were recorded on a gray scale image as a function of the transverse and axial distances. The depth points of 1024 were acquired for the series of images and each image was composed of 512 axial $\times 256$ transverse pixels. Fig. 5 (a) shows the temporal transient intensity profiles of the wavelength-swept laser output, which was measured using an oscilloscope. The electrical intensity profile of the laser output was presented as the sine modulation waveform was applied to the scanning filter. Compared to the similar trace with a SOA gain medium, the output showed relatively higher fluctuations over the original signal profile [7] but the interferogram information can still be induced to convert an OCT image from it. Fig. 5 (b) shows a stable interferogram fringe signal between the reference mirror and sample arm with a mirror from the Mach-Zehnder type OCT.

Figure 6 (a) shows a depth encoded signal using the discrete Fourier transform (DFT) for the sample of the four cover glasses. The two dimensional OCT sample image is also demonstrated in Fig. 6 (b). All four cover glasses and a few air gaps between the cover glasses can be clearly distinguished within the tomography. Fig. 7 shows an OCT image of a human tooth. Compared to the conventional light source for an OCT around the $1300 \mathrm{~nm}, 1000$ or $800 \mathrm{~nm}$ regions, this novel wavelength swept Raman laser around the $1550 \mathrm{~nm}$ region is believed to be useful for specific biological tissues, such as the human tooth, where the dominant loss factor is scattering-induced light attenuation rather than water absorption [14]. The variability in enamel morphology can clearly delineate the junction between the enamel and dentin layers. The inset shows an arrow indicating the scan range of the transverse direction for OCT image. 


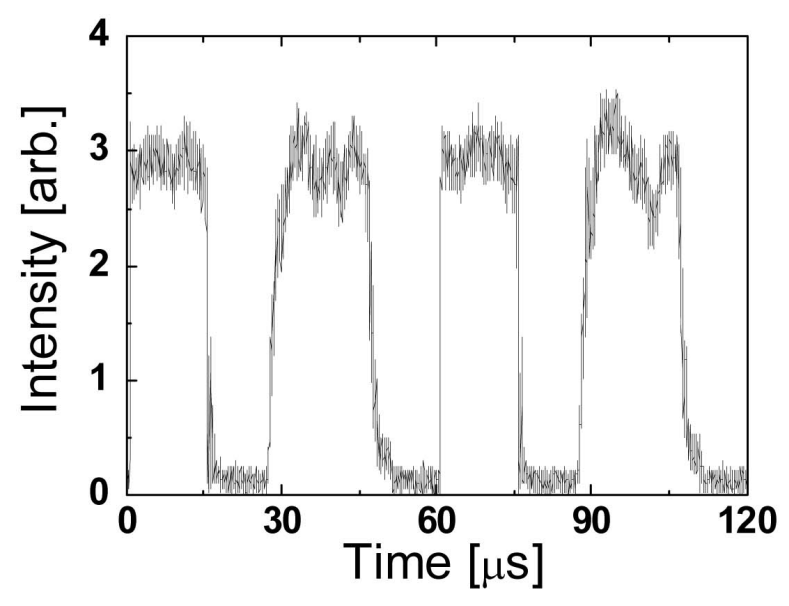

(a)

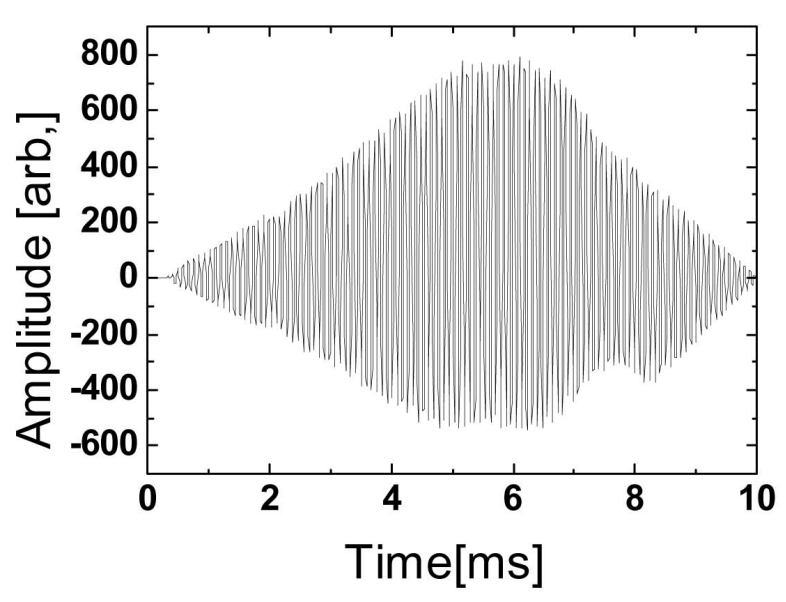

(b)

FIG. 5. (a) Temporal transient intensity profile of wavelength-swept output measured by oscilloscope, (b) Interferogram fringe signal from Mach-Zehnder type OCT with mirror sample.

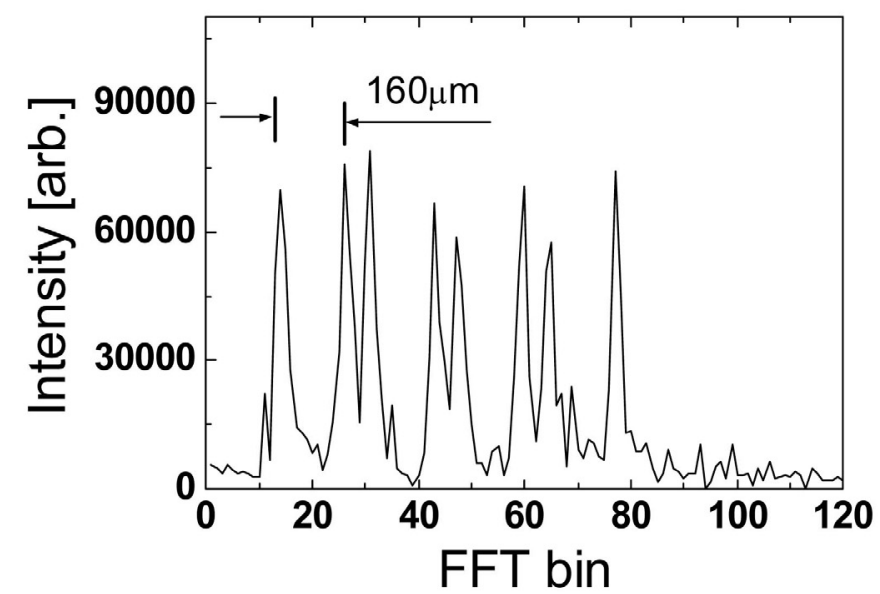

(a)

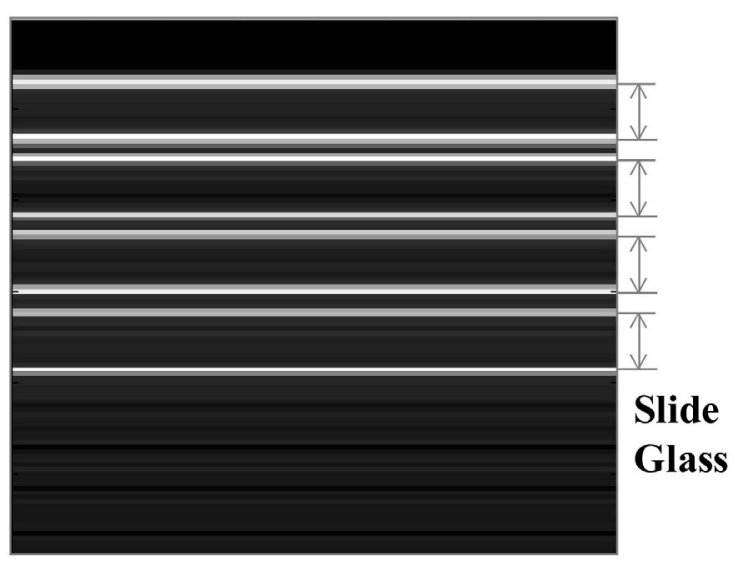

(b)

FIG. 6. (a) Depth encoded signal using Discrete Fourier Transform (b) 2-dimensional OCT image of four slide glasses.

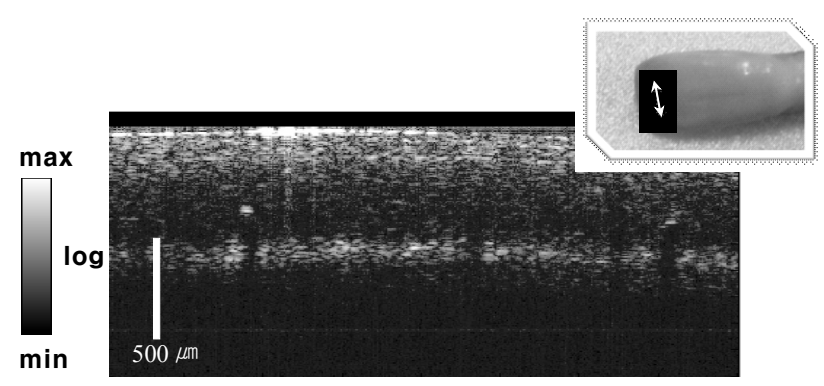

FIG.7. OCT image of a human tooth sample. Inset picture shows the surface location of the transverse scanning of imaging.

\section{CONCLUSION}

This study demonstrated various OCT images using a novel broadband FDML wavelength swept Raman laser. The FDML Raman laser scheme employs the delayed length of an optical fiber as a gain medium, instead of the loss medium in a conventional FDML operation. Spectral broadening of Raman gain was demonstrated experimentally using multi-wavelength pumping laser diodes.

\section{ACKNOWLEDGMENT}

This work was supported by the IT R\&D program of MKE/IITA [2008-F-020-02], Korea.

\section{REFERENCES}

1. S. H. Yun, G. J. Tearney, J. F. de Boer, N. Iftimia, and 
B. E. Bouma, "High-speed optical frequency-domain imaging," Opt. Exp. 11, 2953-2963 (2003).

2. R. Huber, M. Wojtkowski, and J. G. Fujimoto, "Fourier domain mode locking (FDML): a new laser operating regime and applications for optical coherence tomography," Opt. Exp. 14, 3225-3237 (2006).

3. M. Y. Jeon, J. Zhang, Q. Wang, and Z. Chen, "Highspeed and wide bandwidth Fourier domain mode-locking wavelength swept laser with multiple SOAs," Opt. Exp. 16, 2547-2554 (2008).

4. E. J. Jeong, C. S. Kim, M. Y. Jeong, M. K. Kim, M. Y. Jeon, W. Jung, and Z. Chen, "Characterization of FBG sensor interrogation based on a FDML wavelength swept laser,” Opt. Exp. 16, 16552-16560 (2008).

5. J. I. Youn, "Evaluation of morphological changes in degenerative cartilage using $3-\mathrm{D}$ optical coherence tomography," J. Opt. Soc. Korea 12, 98-102 (2008).

6. E. J. Jung, H. S. Lee, J. S. Park, M. Y. Jeong, and C. S. Kim, "Novel wavelength-swept Raman laser for arbitrary gain band OCT,” Proc. SPIE 7168, 716823 (2009).

7. T. Klein, W. Wieser, B. R. Biedermann, C. M. Eigenwillig, G. Palte, and R. Huber, "Raman-pumped Fourier-domain mode-locked laser: analysis of operation and application for optical coherence tomography," Opt. Lett. 33, 2815-2817 (2008).

8. C. S. Kim and J. U. Kang, "Multiplewavelength switching of Raman fiber ring laser incorporating composite polarization-maintaining fiber Lyot-sagnac filter," Appl. Opt. 43, 3151-3157 (2004).

9. S. Namiki and Y. Emori, "Ultrabroad-band Raman amplifiers pumped and gain-equalized by wavelength-divisionmultiplexed high-power laser diodes," IEEE J. Select. Topics Quantum Electron. 7, 3-16 (2001).

10. K. Liu and E. Garmire, "Understanding the formation of the SRS stokes spectrum in fused silica fiber," IEEE J. Quantum Electron. 27, 1022-1030 (1991).

11. H. S. Seo, K. Oh, and U. C. Paek, "Gain optimization of germanosilicate fiber Raman amplifier and its applications in the compensation of Raman-induced crosstalk among wavelength division multiplexing channels," IEEE J. Quantum Electron. 37, 1110-1116 (2001).

12. W. P. Urquhart and P. J. R. Laybourn, "Stimulated Raman scattering in optical fibers with nonconstant losses: a multi wavelength model," Appl. Opt. 25, 25922599 (1986).

13. W. C. Kim and D. W. Park, "Analysis of temperature effects on Raman silicon photonic devices,” J. Opt. Soc. Korea 12, 288-297 (2008).

14. D. Fried, R. E. Glena, J. D. B. Featherstone, and W. Seka, "Nature of light scattering in dental enamel and dentin at visible and near-infrared wavelengths," Appl. Opt. 34, 1278-1285 (1995). 